\title{
Wilhelm Koppe et Josef Oberhauser face à la justice ouest-allemande : exemples d'une première forme de révisionnisme
}

Wilhelm Koppe and Josef Oberhauser versus West-German justice: two examples of a first type of revisionism

Oberhauser versus de West-Duitse justitie: voorbeelden van een eerste vorm van revisionisme

\section{Sila Cehreli}

\section{(2) OpenEdition}

\section{Journals}

Édition électronique

URL : https://journals.openedition.org/temoigner/4162

DOI : 10.4000/temoigner.4162

ISSN : 2506-6390

Traduction(s) :

Wilhelm Koppe en Josef Oberhauser versus de West-Duitse justitie: voorbeelden van een eerste vorm van revisionisme - URL : https://journals.openedition.org/temoigner/4265 [nl]

Éditeur :

Éditions du Centre d'études et de documentation Mémoire d'Auschwitz, Éditions Kimé

Édition imprimée

Date de publication : 2 mai 2016

Pagination : $81-93$

ISSN : 2031-4183

Référence électronique

Sila Cehreli, « Wilhelm Koppe et Josef Oberhauser face à la justice ouest-allemande : exemples d'une première forme de révisionnisme», Témoigner. Entre histoire et mémoire [En ligne], 122 | 2016, mis en ligne le 30 septembre 2021, consulté le 02 février 2022. URL : http://journals.openedition.org/ temoigner/4162 ; DOI : https://doi.org/10.4000/temoigner.4162 


\section{Wilhelm Koppe et Josef Oberhauser face à la justice ouest-allemande : exemples d'une première forme de révisionnisme}

$\rightarrow$ Sila Cehreli

Université de Marmara
(1) Cf. le procès-verbal de l'interrogatoire de Wilhelm Koppe établi à Bonn, les 2 et 3 février 1960. [BArch 162/3243, folios 129 à 167.]

(2) Barch 162/3243, folios 170 à 172 . es criminels nazis qui ont été confrontés à la justice ouest-allemande dès la fin des années 1950 se sont souvent réfugiés dans des stratégies de disculpation. Ces tentatives ont visé à manipuler les faits liés au génocide des Juifs, voire à les dissimuler aux juges d'instruction en l'absence de survivants à une période où l'heure des historiens et des débats mémoriels n’avait pas encore sonné.

La question de la détermination du responsable principal de la «Solution finale» pour le Warthegau / Wartheland en constitue un premier exemple. Qui transmettait les ordres et instructions à l'équipe spéciale de Chełmno, appelée le SS-Sonderkommando Kulmhof? Un rapport adressé, le 12 octobre 1961, par le procureur Dietrich Zeug à la Zentrale Stelle de Ludwigsburg, souligne la complexité de cette question.

Le procureur D. Zeug avait suivi en Israël le procès d'Adolf Eichmann, de début avril à août 1961, en tant qu'observateur du Parquet de Francfort. Lors de son séjour à Jérusalem, il a été abordé, à plusieurs reprises, par l'avocat Dieter W. au sujet de Wilhelm Koppe, le chef supérieur de la SS et de la Police du Warthegau. Ce dernier avait été arrêté, sous le nom de Wilhelm Lohmann ${ }^{1}$, le 31 janvier $1960^{2}$ et attendait l'ouverture de son procès. Le fils de W. Koppe, un collègue de l'avocat Dieter W., préparait la défense de son père pour ce procès qui devait être consacré aux crimes commis à Chełmno.

Wilhelm Koppe a affirmé, au cours d'un de ses interrogatoires, qu'il s'agissait d'un changement de nom légal. Ce changement a été effectué en avril 1945 à Berlin à l'approche certaine de la «catastrophe », à savoir la défaite du III ${ }^{\mathrm{e}}$ Reich, afin de protéger sa famille. Le procureur conduisant l'interrogatoire a, toutefois, attiré l'attention sur le fait que la date et le lieu de naissance du suspect avaient également été falsifiés sur la nouvelle carte d'identité, ce qui n’aurait pas dû se produire dans le cas d'un changement de nom légal. W. Koppe s'est défendu contre cet argument en soulignant la situation chaotique qui régnait au printemps 1945 au ministère de l’Intérieur du Reich, où il avait déposé sa demande. 
Wilhelm Koppe et Josef Oberhauser face à la justice ouest-allemande : exemples d'une première forme de révisionnisme (suite)
(3) Chef de la SS et de la Police de Lublin, Odilo Globocnik a dirigé l'Aktion Reinhard, c'est-àdire la mise à mort des Juifs dans les camps de Betżec, de Sobibór et de Treblinka.

(4) Cf. la déclaration officielle de Dietrich Zeug. [Barch 162/3247, folios 759 à 761 .]

(5) Cf. le procès-verbal de l'interrogatoire de Wilhelm Koppe établi à Bonn, les 2 et 3 février 1960

(6) Le Dr Brandt a fait partie de l'état-major personnel du Reichsführer-SS.

(7) Enst Damzog fut l'inspecteur de la police de sûreté et du service de sécurité du Warthegau.
Dietrich Zeug a écrit dans son rapport officiel, adressé à la Zentrale Stelle de Ludwigsburg, son avis sur une correspondance ayant eu lieu entre l'avocat Dieter W. et le fils de W. Koppe. Cette correspondance lui avait été envoyée par le juge d'instruction du Tribunal de première instance de Bonn, chargé de préparer le procès. On y lit que l'avocat Dieter W. avait demandé à Eichmann si le chef supérieur de la SS et de la Police du Warthegau avait été impliqué dans les crimes commis à Chełmno. Celui-ci aurait répondu, toujours selon cette correspondance, qu'il ne connaissait rien d'une telle implication.

Le procureur Zeug a, cependant, réfuté dans son rapport cette information, suggérant l'innocence de W. Koppe, en se basant sur les investigations qu'il avait réalisées au préalable sur les camps de Bełżec, de Sobibór et de Treblinka. Ce fut à partir de l'étude d'une centaine de témoignages, de nombreux documents trouvés à la fois dans les archives ouest-allemandes et dans les archives internationales, que le procureur a conclu qu'il n'existait aucun indice permettant de placer également le camp de Chełmno sous la responsabilité d'Odilo Globocnik ${ }^{3}$ : une hypothèse avancée par le fils de Wilhelm Koppe dans sa stratégie tentant de prouver l'innocence de son père. D'après les recherches entreprises par D. Zeug, Adolf Eichmann et la section IVB4 du RSHA étaient, cependant, très peu impliqués dans l'Aktion Reinhard, ce qui plaçait Eichmann dans l'impossibilité de livrer des renseignements relatifs à la fonction assurée par Wilhelm Koppe pendant les années de guerre ${ }^{4}$.

Ce dernier a lui-même réfuté, au cours de ses interrogatoires, son implication dans la mise à mort des Juifs à Chełmno, en affirmant qu'il était uniquement chargé de l'accueil des immigrés allemands dans le Warthegau'. Cela étant, il n’a pas nié la réalité de la politique génocidaire. Au contraire, il a élaboré pour son interrogatoire un récit, combinant cette réalité et sa soi-disant innocence.

Selon ce même récit, W. Koppe aurait entendu en 1940 ou 1941 qu'un commissaire de Berlin avait reçu la mission «d'évacuer» les Juifs du Warthegau à l'aide d'un commando, un terme qui, à l'époque, désignait déjà l'extermination physique. Il aurait dû s'agir, à l'origine, d'une opération expérimentale, destinée à tester les travaux préparatoires entrepris avec des gaz toxiques par le Dr Viktor Brack de la chancellerie privée du Führer. Devant cette information qu'il a reçue à la fois du Dr Karl Brandt ${ }^{6}$ et d'Ernst Damzog ${ }^{7}$, W. Koppe avait pensé qu'il ne serait pas question de tuer tous les Juifs, du moins pas ceux qui pouvaient travailler : une affirmation qui ne dénote néanmoins aucune réticence vis-à-vis de l'idée d'assassiner massivement un groupe humain. Cela apparaît de manière encore plus significative, lorsqu'il lui a été reproché, au cours de son interrogatoire, d’avoir adressé, le 3 mai 1942, une correspondance au Dr Karl Brandt au sujet de la soumission des Polonais atteints de la tuberculose à un «traitement spécial» par le Sonderkommando Lange. Sur cette remarque, W. Koppe s'est contenté de répliquer qu'il avait proposé de ne pas tuer ces hommes, mais de les déporter dans le Gouvernement général, parce que 


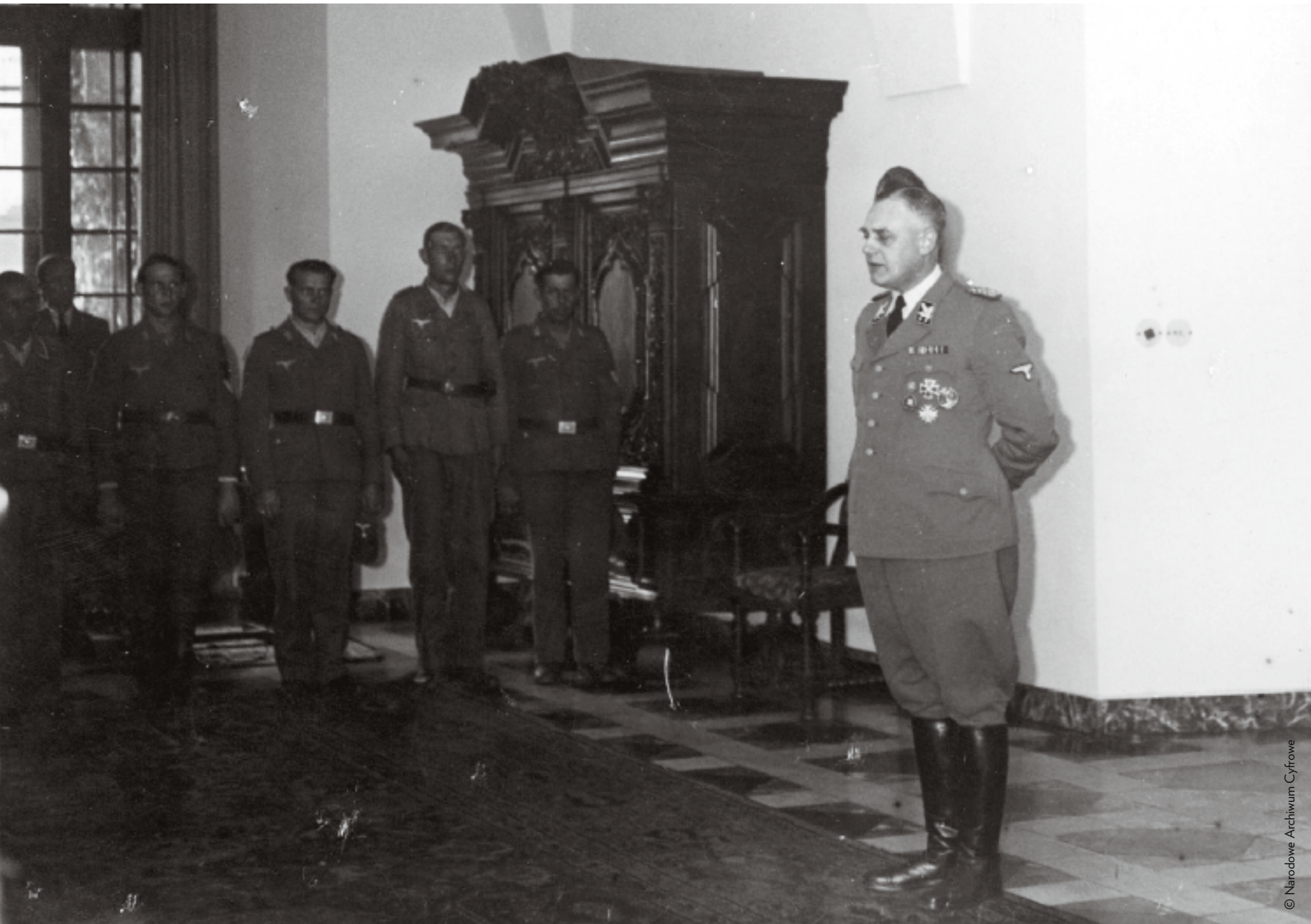

l'exécution des Polonais malades aurait constitué une torture pour les Allemands. Là encore, l'explication de W. Koppe n'exprime aucune forme de condamnation de ce meurtre, ni même une marque de désapprobation sincère.

Après avoir appris la mission meurtrière du Sonderkommando, W. Koppe aurait toutefois tenté - selon son propre récit - de trouver « un moyen intelligent» de contrecarrer la décision prise par le centre ${ }^{8}$. Ainsi, aurait-il contacté Artur Greiser en vue de lui soumettre son plan qui consistait à intégrer les Juifs à l'effort de guerre de l'industrie allemande et donc à prôner de fait le caractère indispensable qu'ils revêtaient pour l'économie, ce qui devait finalement empêcher leur mise à mort. W. Koppe a, par la suite, rapporté qu’Artur Greiser aurait accepté ce «plan » et que tous les deux auraient demandé un rendez-vous à Göring, afin de lui exposer les

_ Le SS-Obergruppenführer Wilhelm Koppe s'adresse aux membres du service de travail allemand au château royal à Cracovie. Juin 1944

(8) Cf. le procès-verbal de l'interrogatoire déjà cité de Wilhelm Koppe. 
Wilhelm Koppe et Josef Oberhauser face à la justice ouest-allemande : exemples d'une première forme de révisionnisme (suite)

\section{(9) Ibid.}

(10) La date donnée par Eichmann s'avère trop tôt. Dans la mesure où il a observé le déroulement d'une opération de gazage, la visite devrait avoir eu lieu un peu plus tard, fin 1941 ou début 1942. détails de leur projet. Arrivés à Berlin, ils auraient été néanmoins reçus par le chef de l'état-major civil de ce dernier, qui leur aurait conseillé d'accomplir la mission qui leur avait été impartie, tout en laissant au Reichsmarschall le souci de l'industrie d'armement. Cela clôt le récit raconté par W. Koppe sur un échec de la soi-disant tentative d'empêcher la mise à mort des Juifs par le Sonderkommando Lange'.

Ce récit, livré par W. Koppe au cours de son interrogatoire à Bonn, nous paraît très douteux, en ce qui concerne notamment son insistance sur le fait que le Sonderkommando aurait reçu ses directives de Berlin et non pas de lui-même. Effectivement, Berlin est bien à l'origine des ordres et instructions liés au projet d'annihilation des Juifs. Mais le poste clef de chef supérieur de la SS et de la Police du Warthegau, fonction exercée du 9 octobre 1939 au 9 novembre 1943 par Wilhelm Koppe, reste l'instance suprême dans la région administrative. C'est le responsable de ce poste qui transmet et diffuse l'ordre, d'une part, aux unités administratives locales concernées et, d'autre part, à l'équipe spéciale chargée d'exécuter la mission meurtrière. La recherche de Ruth Bettina Birn, qui a étudié l'institution du chef supérieur de la SS et de la Police en s'appuyant sur de nombreux exemples concrets, le souligne également. Ainsi, cite-t-elle, à l'appui de son propos, la troisième partie de l'un des premiers décrets attribuant à Erich Von dem Bach-Zelewski le poste de chef supérieur de la SS et de la Police de Breslau. Ce texte marque de manière significative que ce dernier recevrait d'autres missions relevant de sa fonction par «Sonderanweisung (directive spéciale)»(Birn, 1986, p. 11), ce qui montre que ce poste a été conçu, dès sa création, comme un maillon clef qui servirait d'intermédiaire entre le siège de l'État nazi et les fonctionnaires locaux, dont plus tard les exécuteurs de la «Solution finale».

Malgré cela, Adalbert Rückerl affirme dans son étude portant sur l'histoire des centres de mise à mort que l'enquête judiciaire ouest-allemande n'est guère parvenue à préciser par l'intermédiaire de qui et par quelle instance le Sonderkommando avait reçu ses directives (Rückerl, 1979, p. 251). Il a néanmoins noté, lui aussi, qu'un certain nombre d'inspections à Chełmno ont été réalisées par W. Koppe, A. Greiser, E. Damzog et A. Eichmann. Ce dernier a été notamment envoyé au camp «en automne 1941 » par Heinrich Müller, le chef du Bureau IV au RSHA, pour qu'il lui transmette un rapport sur les débuts de l'activité du centre de mise à mort (Von Lang, 1982, p. 71) ${ }^{10}$. Ruth Bettina Birn, quant à elle, a plutôt constaté une certaine dilution dans l'exercice de la fonction de chef supérieur de la SS et de la Police par W. Koppe. Ce dernier, tout en supervisant le centre de mise à mort, aurait toutefois transformé l'office d'Ernst Damzog, l'inspecteur de la police de sécurité, en un bureau de commandement chargé des questions liées à Chełmno (Birn, 1986, p. 181).

Cet argument, énoncé par Ruth Bettina Birn, nous paraît tout à fait possible et compatible avec la structure de Chełmno. Il convient, en effet, de préciser que les autorités administratives de Posen et de Litzmannstadt ont été littéralement 
impliquées dans le fonctionnement du premier centre de mise à mort de la «Solution finale» (Kaczmarek, 2001, p. 29). Il s'agit, notamment, de l'Administration allemande du ghetto de Litzmannstadt placée sous la direction de Hans Biebow. Davantage qu'une seule implication, elles ont été présentes durant le processus de mise à mort qui, contrairement aux autres camps d'extermination, commençait dans le ghetto de Łódź même. Ce constat vaut aussi dans le cas des autres ghettos installés dans les petites villes du Warthegau. Presque aucune sélection n’a eu lieu à Chełmno, ni même lorsqu'il s'est agi de renforcer le groupe des Arbeitsjuden, dont les membres ont été fusillés régulièrement par le $S S$-Sonderkommando. Les camions et trains, partant des ghettos en direction du centre de mise à mort de Chełmno, emmenaient des victimes qui, toutes, étaient acheminées vers les camions à gaz. Lorsque le Sonderkommando souhaitait augmenter l'effectif des Arbeitsjuden, les Juifs qui faisaient partie de ce groupe étaient également majoritairement déportés en camions, du ghetto à Chełmno.

Malgré cette participation active des administrateurs allemands, W. Koppe reste l'autorité locale principale dans l'exécution de la «Solution finale» dans le Warthegau. Un document, portant sa signature et mentionnant le Sonderkommando «qui lui est soumis », en constitue une première preuve. Malgré cette preuve concrète, W. Koppe a tenté, au cours de ses interrogatoires, d'expliquer que ces documents étaient rédigés par des fonctionnaires peu doués et que lui n'avait pas eu toujours le temps d'apporter les corrections nécessaires aux écrits administratifs ${ }^{11}$.

Parallèlement à ce document signé par W. Koppe, un membre du Sonderkommando Kulmhof a affirmé, au cours de son interrogatoire, qu'il avait vu une fois le chef supérieur de la SS et de la Police du Warthegau à Chełmno. Ce membre, qui connaissait bien W. Koppe, ne pouvait se tromper : il avait souvent conduit le chef du Sonderkommando Kulmhof à Posen et à Litzmannstadt, où ce dernier transmettait des rapports relatifs au centre de mise à mort ${ }^{12}$. Un autre garde du Wachkommando, même s’il n'a pu préciser le nom des hauts fonctionnaires ayant inspecté Chełmno, a bien reconnu W. Koppe, après avoir vu sa photo de guerre qui lui a été montrée dans le cadre de son interrogatoire : il a déclaré l'avoir vu soit à Chełmno, soit à Eichstädt, pendant une fête de camaraderie du SS-Sonderkommando ou peut-être encore à une fête d'adieux à Warthbrücken qui avait été organisée à l'occasion de la première liquidation du centre de mise à mort ${ }^{13}$.

Mais la tentative du fils de W. Koppe, qui - en tant que son avocat - a influé sur un témoin en vue de l'amener à changer sa déposition initiale, constitue peutêtre la meilleure preuve de culpabilité du père. Le témoin en question, Oskar K., en sa qualité de général SS, commandant de l'Ordnungspolizei au Warthegau pour la période se situant entre le début de la guerre et la mi-1942, avait auparavant fait une déclaration mettant en cause W. Koppe :
(11) Cf. le procès-verbal de l'interrogatoire de Wilhelm Koppe.

(12) Voir le procès-verbal de l'interrogatoire de Walter Burmeister, un membre du Sonderkommando Kulmhof, établi à Flensburg, le 25 janvier 1961. [BArch 162/3246, folios 631 à 638.] Une décision de 1966 a jugé Wilhelm Koppe inapte à suivre les débats, ce qui a abouti à la suspension des poursuites judiciaires entamées contre lui. Il est décédé huit années après cette décision.

(13) Cf. le procès-verbal de linterrogatoire de Wilhelm Heu, membre du Wachkommando, établi à Meinerzhagen, le 30 novembre et le $1^{\text {er }}$ décembre 1961. [BArch 162/3247, folios 885 à 899.] 
Wilhelm Koppe et Josef Oberhauser face à la justice ouest-allemande : exemples d'une première forme de révisionnisme (suite)
(14) Cf. le procès-verbal de l'audition du témoin Oskar K., général de l'Ordnungspolizei, établi à Starnberg, le 25 mars 1960, par le Bureau du Procureur général à Bonn. [BArch 162/3245, folios 459 à 463 .]

(15) Cf. le procès-verbal de l'audition du témoin Oskar K. établi à Percha, le 30 mai 1960, par le Bureau du Procureur de la République à Bonn. [BArch 162/3245, folios 470 à 472.]

(16) Cf. la déclaration sous serment d'Oskar $\mathrm{K}$ devant le notaire Heribert Thallmair, à Starnberg, le 11 avril 1960. [BArch 162/3245, folios 464 à 467.]
«Tant Koppe qu'également Damzog étaient souvent à Berlin chez Himmler et au RSHA. Les mesures pertinentes, etc. étaient en substance verbalement débattues, les ordres en détail étaient plus tard donnés par Koppe ${ }^{14}$.»

Effectivement, l'agenda professionnel de Himmler témoigne à deux reprises d'un contact de ce type. Le document en question mentionne, d'abord, un entretien qui a eu lieu entre W. Koppe et le Reichsführer-SS, le 4 septembre 1941, de 21 h 30 à $24 \mathrm{~h}$. Cette date précède de deux semaines la décision de déporter les Juifs du Reich, ou plutôt celle de les assassiner. L'heure de l'entretien, ce dernier traitant fort probablement de la nouvelle méthode de mise à mort et de sa première exécution dans le cadre du Warthegau, révèle par ailleurs qu'il s'agit d'une question réservée à un cercle restreint de camarades (Witte, 1999, p. 205).

L'agenda professionnel de Himmler mentionne par ailleurs, pour ce qui est des 16 et 17 avril 1942, une visite du Reichsführer-SS dans le Warthegau, où il a été accueilli par A. Greiser et W. Koppe. Le deuxième jour de cette visite a inclus de 11 h à 12 h une inspection à Warthbrücken et au village de Redern sous le seul guide de W. Koppe. Si le village de Redern, situé à $5 \mathrm{~km}$ de Warthbrücken, représentait pour les autorités nazies un exemple réussi de la politique allemande de colonisation, rien d'autre ne pouvait justifier une visite à Warthbrücken que l'inspection du centre de mise à mort de Chełmno. Nous estimons donc, à l'instar du comité d'historiens ayant préparé la publication de l'agenda professionnel de Himmler, que le nom de Warthbrücken figure sur le programme du 16 avril 1942 en vue de camoufler celui du centre de mise à mort. L'après-midi de cette même journée, confirme notre hypothèse dans la mesure où Himmler a déjeuné encore une fois à 14 h avec le Führer, pour lui transmettre, une heure plus tard, au cours d'une entrevue privée, un rapport sur sa visite au Warthegau (Witte, 1999, p. 398-400), le laboratoire du national-socialisme concernant la concrétisation conjointe de la conquête du Lebensraum pour le peuple allemand et de la mise à mort des Juifs européens.

Oskar K. a cependant signé, le 11 avril 1960, une déclaration sous serment devant un notaire à Starnberg, à la demande du fils de W. Koppe, qui, deux jours plus tôt, lui avait rendu visite à son domicile. Le général a dû, par la suite, rendre compte aux magistrats du Bureau du procureur général de Bonn des raisons de cette déposition supplémentaire. Même s'il a admis alors le fait de ne pas avoir accepté toutes les modifications suggérées par le fils de W. Koppe durant sa déposition devant le notaire, le contenu du témoignage s'en est trouvé considérablement changé15 : « J'ai su plus tard que Heydrich aurait été chargé de la Solution finale de la question juive, et il est possible, que les [SS-]Sonderkommandos étaient son bras prolongé, recevant leurs ordres sans intermédiaire du $\mathrm{RSHA}^{16}$.»

Non seulement cette nouvelle version souscrite par Oskar K. dissimule la véritable fonction de W. Koppe dans le Warthegau, mais elle transforme aussi le vécu du témoin 
en un récit de seconde main. De cette manière, le commandant de l'Ordnungspolizei du Warthegau, qui recevait des ordres de W. Koppe dans la première audition du 25 mars 1960, est devenu, à la suite de la déposition du 11 avril 1960, un acteur situéà l'extérieur de l'organigramme qui n'aurait entendu parler de l'existence de la «Solution finale» que plusieurs années après la guerre.

Si nous examinons, enfin, l'organigramme de l'administration nationale-socialiste élaboré par les magistrats ouest-allemands dans le cadre de l'enquête judiciaire relative à Chełmno, nous pouvons constater que ces derniers ont identifié, en ce qui concerne spécifiquement la région du Warthegau, quatre chaînes de commandement assurant la circulation des ordres et instructions au sein de l'appareil bureaucratique: celles de la police de sûreté, de la police, de l'administration et du RSHA. La fonction de chef supérieur de la SS et de la Police y figure dans la chaîne de commandement de la police de sûreté, où elle a été placée sous l'autorité directe de Himmler, Reichsführer-SS et chef de la police allemande. Le SS exerçant cette fonction demeure, toujours s'agissant de la même chaîne de commandement, le supérieur hiérarchique de l'inspecteur de la police de sécurité et du service de sécurité, ainsi qu’à un niveau inférieur dans l'organigramme, de la police d'État et de la police judiciaire. Seul le Reichsstatthalter (gouverneur du Reich), relevant en principe de la chaîne de com-
_ Fondations du

château utilisé par les

SS Sonderkommando de Chełmno en 1941-1943

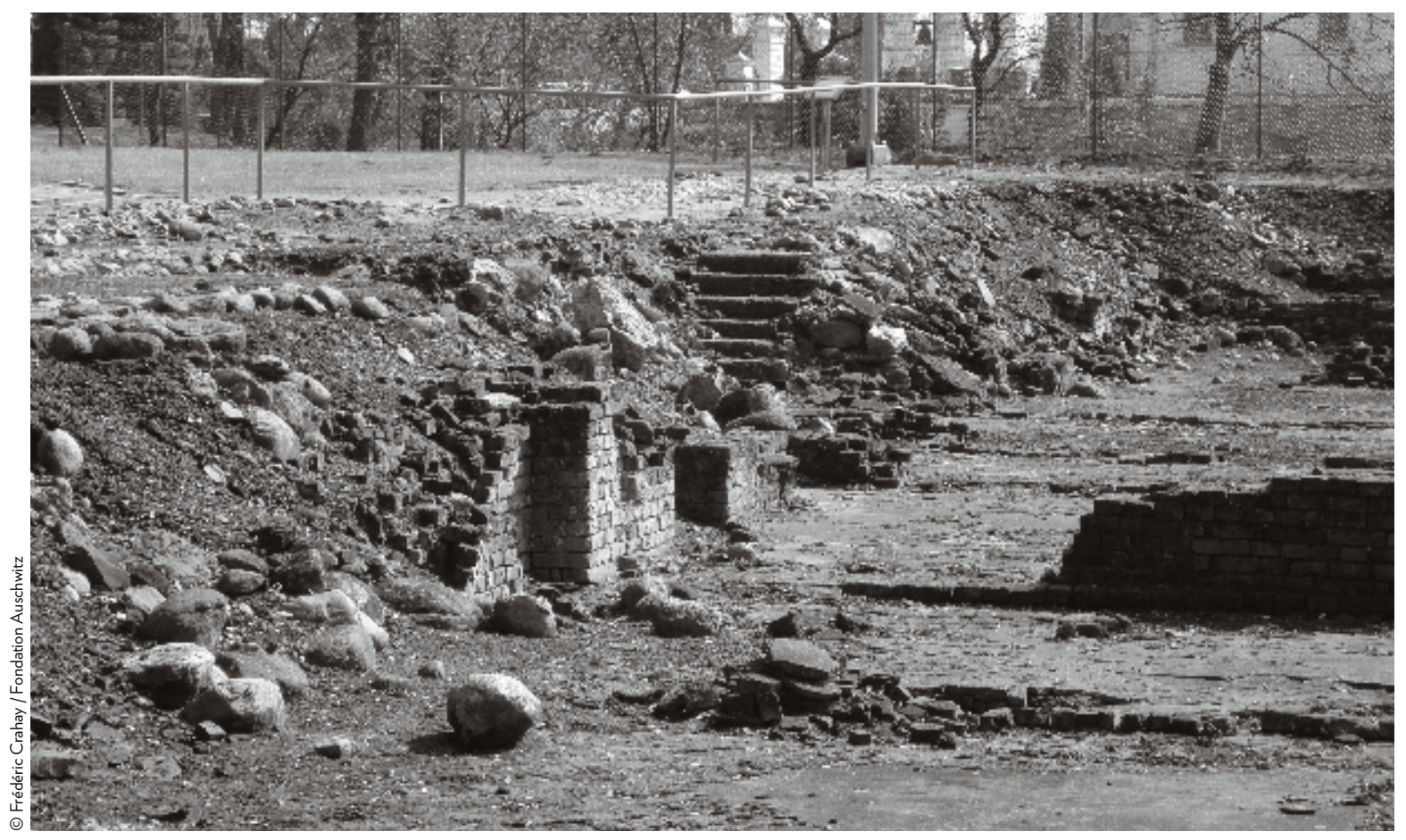




\section{DOSSIER}

Wilhelm Koppe et Josef Oberhauser face à la justice ouest-allemande : exemples d'une première forme de révisionnisme

(suite)

_ Joseph Oberhauser

(17) BA Ludwigsburg, Łódź, 203 AR-Z 69a/59, volume 3, folio 570 . mandement de l'administration et relié directement au gouvernement du Reich, entretient une relation d'interaction avec le bureau du chef supérieur de la SS et de la Police dans la chaîne de commandement du RSHA. Ceci devrait cependant constituer plutôt une exception liée à la personnalité d'Artur Greiser, le gouverneur du Wartheland, qui était particulièrement connu pour son antisémitisme virulent et son engagement de longue date pour la cause nationale-socialiste ${ }^{17}$.

La difficulté réside donc dans la confusion créée par ces différentes chaînes de commandement et sur cette question de la circulation de l'information au sein de la bureaucratie du III ${ }^{\mathrm{e}}$ Reich. Comment expliquer, en effet, que le RSHA, placé à l'instar du chef supérieur de la SS et de la Police sous l'autorité directe de Himmler, n'entretienne aucune relation avec cette instance bien qu'il apparaisse dans la chaîne de commandement de la police de sécurité comme le supérieur hiérarchique de l'inspecteur de la police de sécurité, de la police d'État et de la police criminelle? Le RSHA s'occupe, en réalité, essentiellement des questions techniques liées à l'utilisation des camions à gaz dans le Warthegau, tandis que le chef supérieur de la SS et de la Police représente Himmler dans la région : il transmet - sans intermédiaire - ordres et instructions, tout en supervisant leur exécution.

Josef Oberhauser, qui a accompagné Christian Wirth tout au long de sa mission meurtrière dans le cadre de «l'Opération Reinhard», présente un deuxième exemple de stratégie révisionniste formulée devant un juge d'instruction. Alors que C. Wirth dirigeait le camp de Bełżec, Josef Oberhauser accomplissait déjà, à ses côtés, sa tâche en tant que représentant du commandant. De même, après la nomination du premier à la fonction d'inspecteur des SS-Sonderkommandos de «l'Opération Reinhard », il l'a suivi à Lublin pour y devenir son aide de camp. 


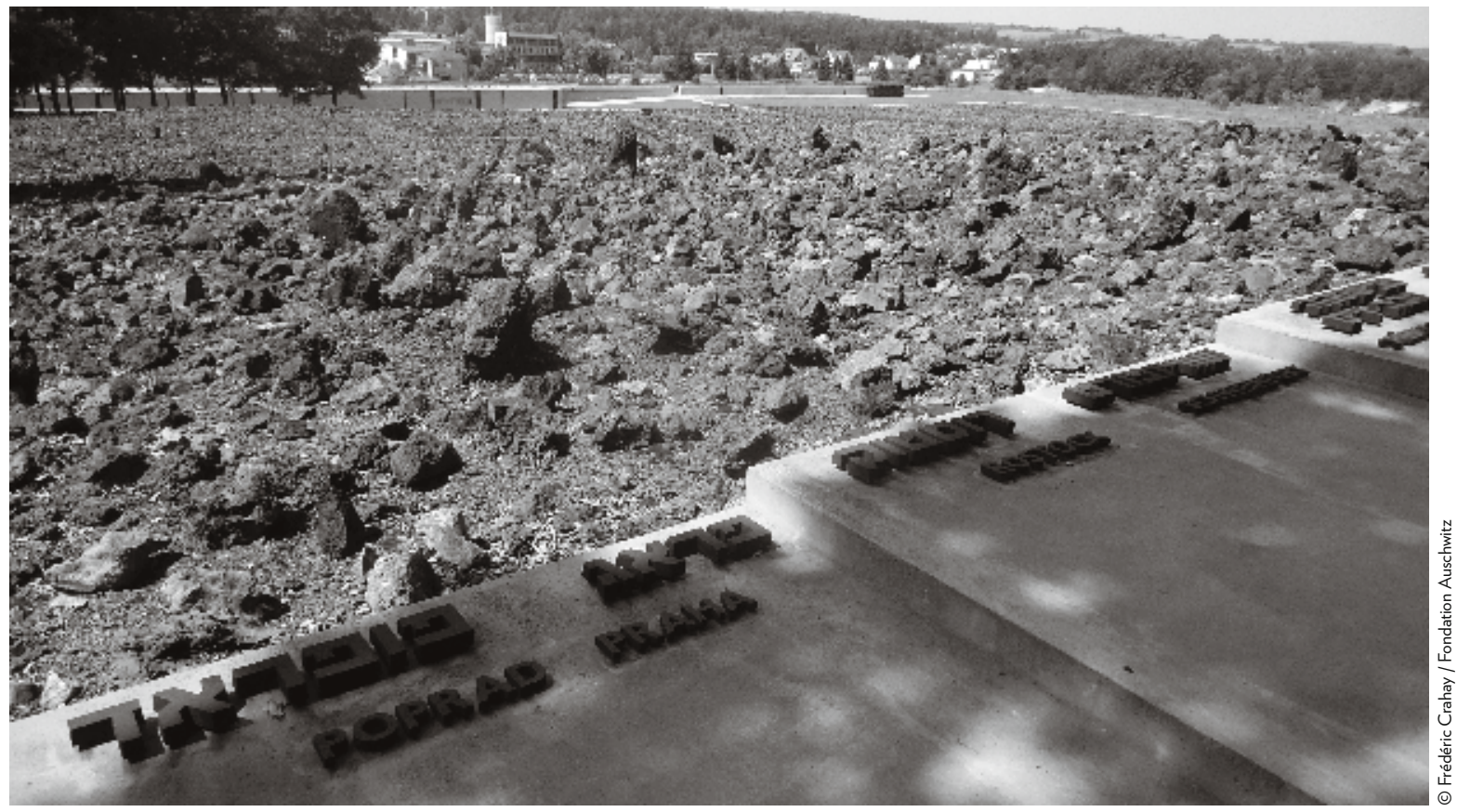

Né en 1915 à Munich, Josef Oberhauser a commencé à travailler comme ouvrier agricole après avoir terminé les huit années de la Volksschule. Après son service militaire, qu’il a accompli de 1934 à 1935, il s'est déclaré volontaire pour la SS-Verfügungstruppe, d'où il a été intégré peu avant la guerre à la Leibstandarte Adolf Hitler à Berlin-Lichterfelde. L'unité dont il faisait partie avait participé à la campagne de Pologne. Mais son implication dans la politique d'extermination nazie commence avec son affectation, vers Noël 1939, à Grafeneckl'un des établissements d'euthanasie. Josef Oberhauser y est resté environ trois mois ; puis il a été envoyé par la T4 aux instituts de Brandebourg et de Bernburg. À son affectation au programme d'euthanasie a succédé sa mission dans le cadre de «l'Opération Reinhard ». Transféré dès l'automne 1941 au Bureau du Chef de la SS et de la Police de Lublin, il compte parmi les premiers membres de la nouvelle équipe spéciale ${ }^{18}$.

Àla fin de la guerre, il a été arrêté par les troupes britanniques et mis en détention dans un camp de prisonniers de guerre, d'où il est toutefois parvenu à s'échapper. Il a été pris, une deuxième fois, en 1948, par les Russes, alors qu'il essayait de passer clandestinement de la zone d'occupation américaine à celle de l'Union soviétique en vue de rendre visite à un camarade de guerre.

Josef Oberhauser a été condamné le 24 septembre 1948 par le Tribunal de grande instance de Magdebourg à quinze années de réclusion criminelle. Cette peine a été prononcée par les Russes à cause de sa participation au programme d'euthanasie, de son appartenance à la Waffen-SS, ainsi que de ses activités contre les partisans
_ Le mémorial de Betżec actuel, inauguré en 2004, recouvre l'entièreté de l'ancien centre d'extermination.

(18) Cf. le procès-verbal de l'interrogatoire de Josef Oberhauser établi à Munich, le 15 septembre 1960, par le Bureau régional de la police judiciaire bavaroise. [BArch 162/3169, folios 1036 à 1040.] 
Wilhelm Koppe et Josef Oberhauser face à la justice ouest-allemande : exemples d'une première forme de révisionnisme (suite)
(19) Cf. le procès-verbal de l'interrogatoire de Josef Oberhauser établi à Munich, le 26 février 1960, par le Parquet près du Tribunal de grande instance de Munich I. [BArch $162 / 3167$, volume 4 , folios 656 à 660.]

(20) lbid.

(21) Cf. le procès-verbal de l'interrogatoire de Josef Oberhauser établi à Munich, le 20 avril 1960, par le juge d'instruction près du Tribunal de grande instance de Munich I. [BArch 162/3167, folios 763 à 765 .] russes et polonais : il n’a été alors aucunement question de son implication dans les crimes commis dans le cadre de «l'Opération Reinhard». Cependant, à l'instar d'autres prisonniers de guerre allemands placés en détention en Union soviétique, il a été amnistié en avril 1956, avant même d'avoir complètement purgé sa peine. Il s'agit, en fait, d'une décision prise par les Russes à l'occasion d'une visite de Konrad Adenauer à Moscou' ${ }^{19}$.

Une fois rentré en Allemagne fédérale, Josef Oberhauser s’est installé à Munich, où il a commencé à travailler en tant que serveur dans une brasserie jusqu’à son arrestation en février 1960, dans le cadre de l'enquête judiciaire investiguant sur les crimes nazis commis au camp de Bełżec ${ }^{20}$.

Au début de ses interrogatoires, Josef Oberhauser a catégoriquement nié son appartenance à l'équipe de «l'Opération Reinhard». Selon sa première version des faits, il a présenté sa fonction comme une tâche se limitant à l'entraînement des unités composées d’Ukrainiens et de Volksdeutsche. Même s'il a reconnu avoir connu l'existence du camp de Bełżec, il a affirmé qu'il ne s'y était jamais rendu. Il aurait accompagné, une seule fois, le transport d'une trentaine d'anciennes baraques de la Wehrmacht jusqu'au portail de ce camp, sans toutefois y entrer. D’après son récit, il n’aurait pas été autorisé à pénétrer dans l'espace rectangulaire strictement isolé du site de mise à mort dont la topographie incluait, à l'encontre d'autres camps d'extermination, également quelques lieux ordinaires du village de Bełżec.

Dans un autre interrogatoire réalisé deux mois après le premier, Josef Oberhauser a rectifié son récit. Il a alors reconnu être entré à deux reprises dans le camp de Bełżec, mais en soulignant que cela s'était passé avant la mise en marche de l'activité meurtrière du camp. La raison de ce changement peut, sans aucun doute, être expliquée par l'existence de dépositions concordantes d'anciens membres de SS-Sonderkommandos ayant mentionné la présence de Josef Oberhauser à Bełżec. Ce dernier a dû, par conséquent, réadapter son récit en justifiant ses séjours dans le camp par des missions ponctuelles : la première d'entre elles, qui lui aurait été confiée par le Bureau du Chef de la SS et de la Police de Lublin, aurait consisté, en décembre 1941, à acheminer dix jours d'approvisionnement à l'intention de l'équipe des gardes ukrainiens de Bełżec. Il aurait effectué, ensuite, son second séjour dans ce camp aux environs de février - début mars 1942. Cette fois, il aurait été chargé de conduire à Bełżec un contingent de 30 gardes ukrainiens et de livrer une autre quantité de dix jours d'approvisionnement ${ }^{21}$.

À la suite de ces premiers interrogatoires et surtout du fait de l’absence de dépositions recueillies auprès des survivants de Bełżec, les magistrats ouest-allemands ne sont pas parvenus à constituer un dossier d'instruction susceptible d'ouvrir la voie à un procès. Par conséquent, Josef Oberhauser a été libéré en août 1960 de la prison de Munich-Neudeck, où il était détenu préventivement depuis février 1960. 
Il a pu alors reprendre son travail de serveur dans la même brasserie. Il existe une courte scène dans Shoah qui montre Josef Oberhauser en train de travailler dans cette brasserie. Ce dernier a, cependant, préféré garder le silence, alors qu'il était confronté aux questions de Claude Lanzmann lui demandant s'il se souvenait de Bełżec.

Ce n'est qu'en décembre 1962 que Josef Oberhauser a enfin admis qu'il avait servi au camp de Bełżec. Mais son récit était toujours construit en décalage avec la réalité. Selon ce récit, si celui-ci était, à partir de Noël 1941, effectivement un subordonné de Christian Wirth, le commandant du centre de mise à mort, sa fonction aurait uniquement consisté à assurer le contact entre ce dernier et le Bureau de Globocnik. Cela signifierait que sa mission se serait seulement limitée à du transfert de matériel
_ Le site de la forêt à

Rzuchów près de Chełmno. Le mémorial a été

réorganisé en 2015, tenant compte des résultats de la recherche actuelle.

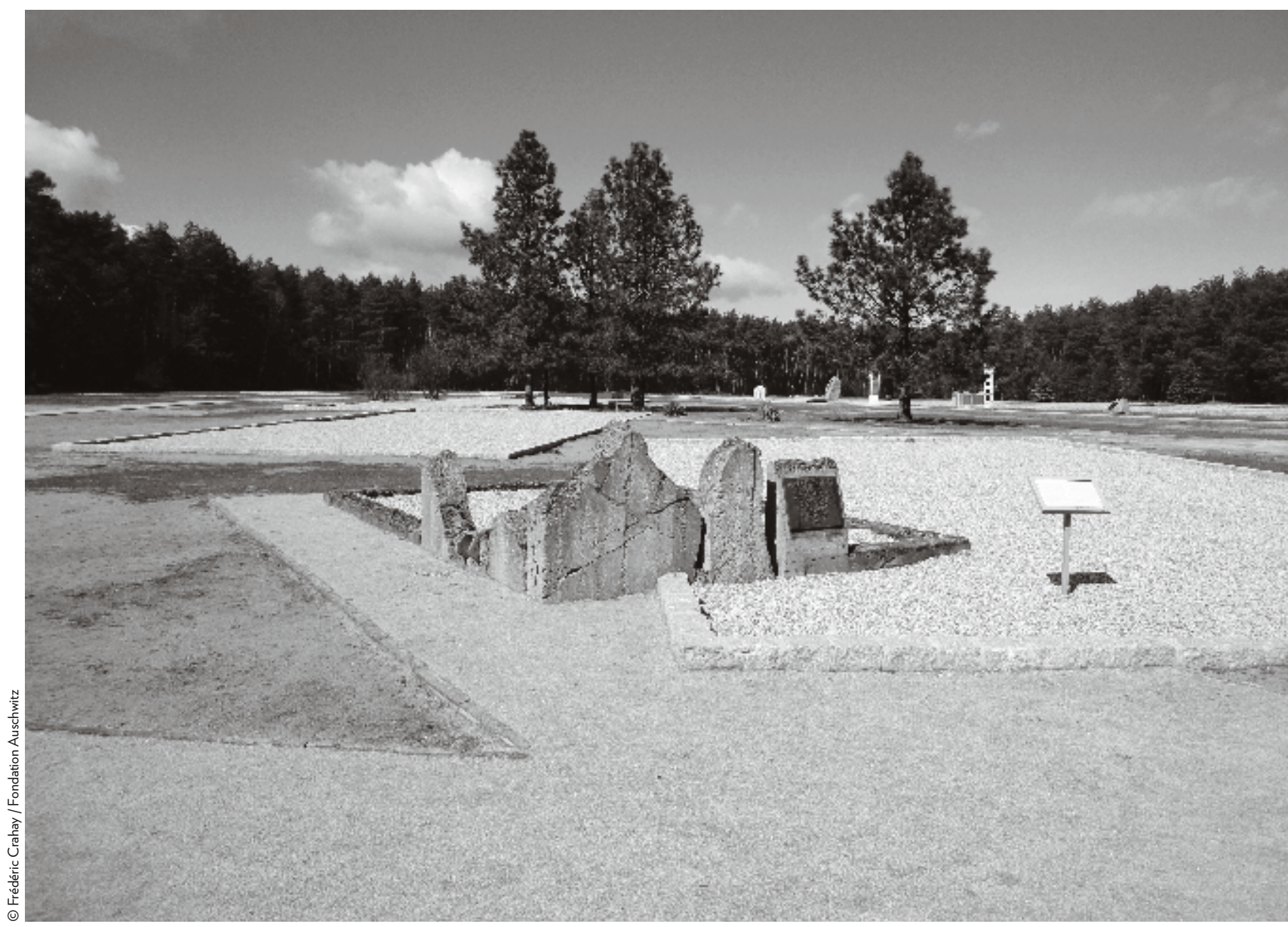


Wilhelm Koppe et Josef Oberhauser face à la justice ouest-allemande : exemples d'une première forme de révisionnisme (suite)
(22) Cf. le procès-verbal de l'interrogatoire de Josef Oberhauser établi à Munich, les 12 et 14 décembre 1962, par le premier procureur. [BArch 162/3172, folios 1678 à 1688.]

(23) Cf. le procès-verbal de l'interrogatoire de Josef Oberhauser établi à Munich, le 15 septembre 1960.

(24) Cf. le procès-verbal de l'interrogatoire de Willy Häusler établi à Bremerhaven, le 28 novembre 1962, par le Parquet près du Tribunal de grande instance de Munich I. [BArch 162/3171, folios 1655 à 1659.]

(25) Cf. le procès-verbal de l'interrogatoire de Karl Schluch établi à Kleve, les 10, 11 et 12 novembre 1961, par le Bureau régional de la police judiciaire bavaroise. [BArch 162/3171, folios 1503 à 1525.] de construction entre Lublin et Bełżec, de même qu’à l'encadrement des équipes de gardes ukrainiens. Toujours selon ce récit discutable, la fonction de représentant de Christian Wirth, une fonction qui lui a été attribuée par plusieurs anciens membres de $S S$-Sonderkommandos, aurait été en réalité exercée par Gottfried Schwarz. Ce dernier était, cependant, le représentant de Gottlieb Hering, le deuxième commandant de Bełżec. Si Josef Oberhauser a reconnu le fait d'avoir quelquefois donné des ordres au personnel allemand de ce centre de mise à mort, cela ne se serait produit que sur l'instruction explicite de Christian Wirth ${ }^{22}$.

Josef Oberhauser a transmis, par ailleurs, des informations falsifiées sur les différentes périodes du camp de Bełżec. Il a, plus précisément, affirmé qu'il avait été chargé, à l'automn 1941, par le Bureau de Globocnik, d'une part, de ramasser dans le village de Bełżec le matériau de guerre - qui se trouvait dispersé sur le terrain le long de l'ancienne ligne de démarcation germano-soviétique - et, d'autre part, d'expédier ce matériau abandonnéà Lublin. Il est fort probable que Josef Oberhauser ait effectivement reçu l'ordre d'exécuter une telle mission. Mais celle-ci n'a aucunement duré, comme il l’a suggéré dans un de ses interrogatoires, jusqu’à l'été 1942 : une période qui - toujours selon son propre récit - marque le début de sa prétendue affectation en tant que garde aux usines d'armement allemandes à Lublin. Josef Oberhauser était, cependant, désigné à cette époque aide de camp de Christian Wirth, nommé inspecteur des SS-Sonderkommandos de «l'Opération Reinhard», ce qu'il n'a pourtant pas reconnu. Lorsque les magistrats ouest-allemands ont établi, sur la base d'interrogatoires d'anciens membres des SS-Sonderkommandos, sa participation, à la fin de l'été 1942, à une visite de Globocnik à Treblinka, Josef Oberhauser s'est encore une fois défendu en expliquant qu’il avait alors uniquement été assigné à la commission d'inspection en tant que simple escorte ${ }^{23}$.

Des indices confirmant l'implication directe de celui-ci dans «l'Opération Reinhard » n'ont toutefois pas manqué. Willy Häusler, un membre du Bureau de Lublin, a notamment précisé qu'il devait rassembler les correspondances administratives dans un dossier pour les soumettre à la signature de Josef Oberhauser en l'absence de Christian Wirth : la signature valant pour les magistrats ouest-allemands l'exercice d'un pouvoir de décision et d'une autorité de donner des ordres ${ }^{24}$. Mais, même en l'absence de ce pouvoir, le fait d'appartenir au personnel de Bełżec suffit largement - comme le souligne Karl Schluch, un membre de cette équipe - à démontrer la culpabilité d'un suspect : «Si aucun d'entre nous ne peut être blanchi, alors Josef Oberhauser le peut bien moins encore [que quiconque]... Au fond, le camp de Bełżec servait, tout de même, uniquement à un seul but et que d'autre aurait donc fait Oberhauser là-bas et pour quelle raison aurait-il été promu ${ }^{25}$ ?»

Ce ne fut qu'en décembre 1962 que Josef Oberhauser a enfin reconnu, au cours d'un nouvel interrogatoire, avoir fourni des informations falsifiées sur sa fonction pendant les années de guerre. Il a justifié son attitude par sa conviction que, parmi 
les membres de l'Einsatz Reinhard toujours en vie, il avait, lui, le grade le plus élevé. Il avait alors pensé qu'une participation même relative à cette opération l'aurait rendu responsable de toute l'entreprise meurtrière ${ }^{26}$.

Ces deux exemples mentionnés brièvement ci-dessus soulignent le fait que le témoignage de seulement cinq rescapés - trois de Chełmno et deux de Bełżec - n’ont pas permis aux anciens membres des SS-Sonderkommandos de maintenir leurversion falsifiée des années d'occupation nazie en Pologne. Ce faisant, ces témoignages ont conduit les suspects à se dénoncer eux-mêmes, ce qui a également contribuéà l’avancement des difficiles enquêtes préliminaires ouest-allemandes. Mais le témoignage de rares rescapés a aussi et surtout fait évoluer la version falsifiée des suspects, d'un négationnisme absolu vers un révisionnisme conciliant la réalité du génocide avec une stratégie de disculpation.

\section{BIBLIOGRAPHIE}

- Birn, Ruth Bettina (1986) : Die Höheren SS- und Polizeiführer. Himmlers Vertreter im Reich und in den besetzten Gebieten ("Les chefs supérieurs de la SS et de la Police. Les représentants de Himmler dans le Reich et dans les territoires occupés »), Düsseldorf, Droste Verlag.

- Kaczmarek, Marian (2001) : « Die nationalsozialistischen Pläne für die Vernichtung der Juden im "Reichsgau Wartheland" ("Les plans nationaux-socialistes pour l'extermination des Juifs au "Reichsgau Wartheland" ») » in Struck, Manfred (dir.), Chełmno/Kulmhof. Ein vergessener Ort des Holocaust? (Chełmno/Kulmhof. Un lieu oublié de l'Holocauste?), Bonn/Berlin, Gegen Vergessen - Für Demokratie, p. 15-30.

- Rückerl, Adalbert (1979) : Nationalsozialistische Vernichtungslager im Spiegel deutscher Strafprozesse. Belzec, Sobibor, Treblinka, Chełmno ("Les camps d'extermination nationaux-socialistes à travers le miroir des procès pénaux allemands. Bełżec, Sobibór, Treblinka, Chełmno »), Munich, Deutscher Taschenbuch Verlag.

- Von Lang, Jochen (1982) : Das Eichmann-Protokoll. Tonbandaufzeichnungen der israelischen Verhöre ("Le procès-verbal d'Eichmann. Enregistrements des interrogatoires israéliens »), Berlin, Severin und Siedler.

- Witte, Peter (1999) : Der Dienstkalender Heinrich Himmlers 1941/42 («L'agenda professionnel d’Heinrich Himmler 1941/42 »), Hambourg, Hans Christians Verlag.

(26) Cf. le procès-verbal de l'interrogatoire de Josef Oberhauser établi à Munich, le 13 décembre 1962, par le premier procureur. [BArch 162/3172, folios 1689 à 1693.$]$ 